\title{
Position Estimation of Mobile Robots using Multiple Active Sensors with Network
}

\author{
TaeSeok Jin* \\ ${ }^{*}$ Dept. of Mechatronics Engineering, Dongseo University, Busan, 617-716, Korea
}

\begin{abstract}
Recently, with the development of service robots and the concept of ubiquitous, the position estimation of mobile objects has received great interest. Some of the localization schemes are introduced, which provide the relative location of the moving objects subjected to accumulated errors. To implement a real time localization system, a new absolute position estimation method for a mobile robot in indoor environment is proposed. Design and implementation of the localization system comes from the usage of active beacon systems (based upon RFID technology). The active beacon system is composed of an RFID receiver and an ultra-sonic transmitter. The RFID receiver gets the synchronization signal from the mobile robot and the ultra-sonic transmitter sends out the traveling signal to be used for measuring the distance. Position of a mobile robot in a three dimensional space can be calculated basically from the distance information from three beacons and the absolute position information of the beacons themselves. In some case, the mobile robot can acquire the ultrasonic signals from only one or two beacons, due to the obstacles located along the moving path. In this paper, a position estimation scheme using fewer than three sensors is developed. Also, the extended Kalman filter algorithm is applied for the improvement of position estimation accuracy of the mobile robot.
\end{abstract}

Key Words: Position, Estimation, mobile robot, Ultrasonic sensor, Network.

\section{Introduction}

In the near future, Through Ubiquitous computing and Ubiquitous network, various service newly may be invented. Specially, it is basic and one of important technology that recognize position of robot in environment that provide service that man and robot systems recognize position of same object always[1]. To works something for home robot or service robot in some surrounding, position and recognition are very important elements. Before, robot had been used mainly to process business in limited working space. But Mobile robot has wide application in industry field and home. They are especially necessary for tasks difficult and dangerous for men to perform. Therefore, It is necessary to find confidence position of present robot in case give orders to mobile robot in some surrounding. Position measurement is divided by outdoor and indoor, There are relativity position measurement method and absolute position measurement method. Relativity position recognition base on dead reckoning representatively.

Dead reckoning mainly calculates its position with data acquired from a rotary encoder, which is corrected to wheel. It is simple, low-cost, and calculate position easy by real time. But, It won't receive correct position because of slippage, a rough surface, and sensor error[2].

Generally absolute position recognition method uses navigation beacons, active or passive landmarks, map matching

Manuscript received Jul. 7, 2011; revised Nov. 2, 2011; accepted Nov. 7;

* Authors: jints@dongseo.ac.kr or satellite based navigation signals. GPS(Global Positioning System)[3] is representative one of method to solve problems of relativity position recognition method. But, GPS can be used in only outdoor environment and the position error is oversized to apply to Mobile robot. Usually, ultra-sonic sensor had been used extensively to system to recognize position of robot in indoor environment.

Used ultrasonic in [4] to measurement equipment to measure position and direction of robot. This system can measure distance information at the same time in 3 firm places

It sends 3 ultrasonic by different frequency for these processing and 3 reply telegram receivers are threaded to robot and controlled. Position and direction angle of robot are decided from geometrical parameter of beacon that is acquired from ultrasonic scan data. This system cannot do real time calculate. Because robot must stop and do scan to recognize position of robot.

In the meantime, ultra-sonic way to install ultrasonic generator to transfer space in [5] proposed, robust against noises and fast calculation because process direct wave that is shot from ultrasonic generator unlike other ways to process reflected wave. But, there is burden to install controller that is linked with each ultrasonic generators and there is problem that cannot control each ultrasonic generators according to situation because only receive ultra-sonic in mobile robot.

To overcome these problems proposed simple radio frequency (RF : Radio Frequency) radio, Global ultra-sonic sensor system of GPS (Global Positioning System) structure that use ultra-sonic sensor in the latest research about position recognition of robot [10]. But, It have some problems to use this system in actual systems, because there are suburbs of a town city time-invarying obstacles of human and desk and 
chair in public place or general home that mobile robot is space that exist problem that do not recognize position of mobile robot happens.

This research suggests ABS (Active Beacon Sensor) System that use Twin Beacon Detection algorithm and Linear Incremental algorithm to supplement problem of system that explain before. When there are obstacles, Position conclusion uses ultra-sonic general triangulation technique to take advantage of RFID application technology basically and use Twin Beacon Detection or Linear Incremental that is a robust algorithm against noises. Also, used extended Kalman filter to remove noise of measured signal. Composition of this paper is as following.

First, This paper briefly introduces extension Kalman filter algorithm for own position conclusion of mobile robot under ABS (Active Beacon Sensor) System's composition and system that see in Chapter 2, and explain about robust algorithm about outside environment or noise that propose in chapter 3, and present computer simulation and experiment result to efficiency of system that see in chapter 4 and concluded conclusion in chapter 5 .

\section{Active Beacon Sensor(ABS) System}

\subsection{System structure}

The proposed localization method is developed for a mobile robot, which would be used as Humanoid robot: A robot that helps the human in different tasks inside the room. The environment is the indoor room, which is assumed to have a two-dimensional floor plane and composed of walls and corners. This room also contains different objects like desks, tables, computers and other objects.

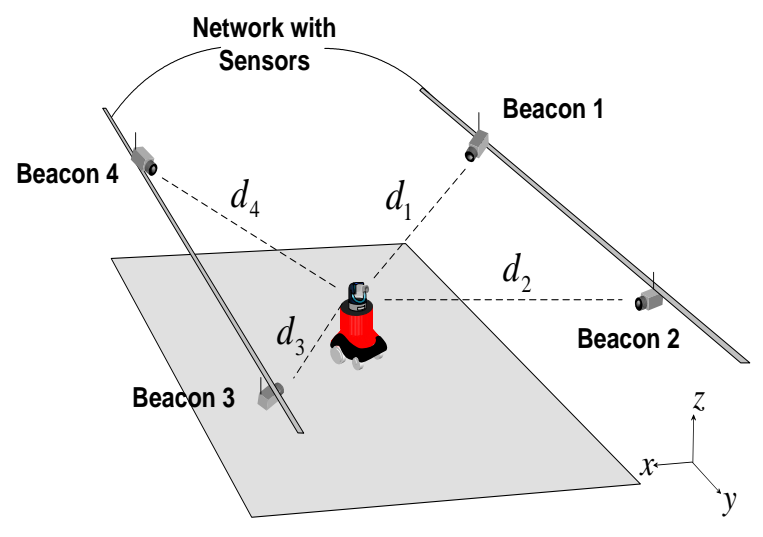

Fig. 1. Structure of the ABS system.

Ultrasonic generator was placed in fixed position in motion space of a mobile robot. The number of ultrasonic generator installed being more than minimum 3 and considers ultrasonic direction and arrival area. Four beacons are installed on the ceiling corners of a block as shown in Fig. 1.

\subsection{Principle of measurement using ultrasonic}

This section describes principal of basic measurement using ultrasonic waves. The proposed measuring system basically consist of one receiver module which is mounted on the mobile robot and four transmitter modules (Beacon in our system). The receiver module consists of RF-transmitter and can produce a burst of signal at the periodically cycle and necessary circuit for detection and amplification of ultrasonic waves. The beacon consists of ultrasonic sensor.

When the receiver module sends RF signals, it starts a counter at the receiving circuit. The beacon receives the RF signal and then almost at the same time sends ultrasonic wave to the receiver module (the time of flight of RF signal is negligible and the delay of detecting circuit is considered as a fix value in calculations). This counter will continue to count until the reception of ultrasonic signal. The value of this counter is proportional to the time of flight (TOF) of ultrasonic wave and can be used to calculate the distance between receiver and beacon:

$$
\begin{aligned}
& v=331.5+0.6 \times t[\mathrm{~m} / \mathrm{sec}] \\
& s=n \times f-t_{d} \\
& r[m]=v[\mathrm{~m} / \mathrm{sec}] \cdot s[\mathrm{sec}]
\end{aligned}
$$

where $T$ is the temperature $\left({ }^{\circ} c\right), f$ is the counter clock rate, $n$ is the timer count, $t_{d}$ is the delay of detection circuit, $s$ is the total time of flight of wave, $v$ is velocity of wave and $r$ is the range data.

The range $r$ to any given beacon is projected to the plane of receiver $d$, which maps out a circular locus of possible positions. Using the three distance information $d_{1}, d_{2}, d_{3}$ and location information $\left(x_{1}, y_{1}\right),\left(x_{2}, y_{2}\right),\left(x_{3}, y_{3}\right)$, the location of the mobile robot $\left(x_{r}, y_{r}\right)$ can be obtained as follows:

$$
\left[\begin{array}{c}
\left(x_{r}-x_{1}\right)^{2}+\left(y_{r}-y_{1}\right)^{2} \\
\left(x_{r}-x_{2}\right)^{2}+\left(y_{r}-y_{2}\right)^{2} \\
\left(x_{r}-x_{3}\right)^{2}+\left(y_{r}-y_{3}\right)^{2}
\end{array}\right]=\left[\begin{array}{l}
d_{1}^{2} \\
d_{2}^{2} \\
d_{3}^{2}
\end{array}\right]
$$

\subsection{Position Estimation Using EKF}

If a noise is reported in a measured signal, we can use a Kalman filter in order to estimate a true value of a signal[6]-[8]. In this paper, nonlinear relation is between measurement distance signal and global coordination value of a mobile robot in ABS system. Therefore, we apply an extended Kalman filter for a nonlinear system in this system [9]-[10]. Mobile robot state vector $r$ consists of the 2 dimensional position $(x, y)$ and heading angle $\theta$. And, state equation can be written from point model as follows. 


$$
\begin{aligned}
& r_{f, k+1}=f\left(r_{f, k}, u_{k}, q_{k}\right) \\
& =\left\{\begin{array}{c}
{\left[\begin{array}{c}
x_{f, k}+T v_{k} \cos \theta_{k}+q_{1, k} \\
y_{f, k}+T v_{k} \sin \theta_{k}+q_{2, k}
\end{array}\right] \quad \text { if } w_{k}=0} \\
{\left[\begin{array}{c}
x_{f, k}+\left(-l \cos \theta_{k}+\rho_{k} \sin \theta_{k}\right)\left(1-\cos \left(T w_{k}\right)\right) \\
-\left(l \sin \theta_{k}+\rho_{k} \cos \theta_{k}\right) \sin \left(T w_{k}\right)+q_{1, k} \\
y_{f, k}-\left(l \sin \theta_{k}+\rho_{k} \cos \theta_{k}\right)\left(1-\cos \left(T w_{k}\right)\right) \\
+\left(l \cos \theta_{k}+\rho_{k} \sin \theta_{k}\right) \sin \left(T w_{k}\right)+q_{2, k}
\end{array}\right] \text { if } w_{k} \neq 0}
\end{array}\right.
\end{aligned}
$$

where, $r=[x, y]^{t}$ is state vectors, $u=[T, \Delta \theta]^{t}$ is control inputs and $w_{k}=\left[w_{1, k}, w_{2, k}, w_{3, k}\right]^{t}$ is Gaussian noise with the mean value 0 and dispersion $\mathrm{Q}$, that is, $w_{k} \sim N(0, Q)$, the following subscript $k$ means time.

Now, the ultrasonic observation equation is as follows in a position of ultrasonic receiver sensor, $p_{f}$ and $p_{g}$.

$$
z_{k}=h\left(r_{k}, v_{k}\right)
$$

where, the measurement noise, $v_{k}=\left[v_{1, k}, v_{2, k}\right]^{t}$ is Gaussian, that is $v_{k} \sim N(0, G)$ and function $h\left(r_{k}, v_{k}\right)$ is as follow:

$$
h\left(r_{k}, v_{k}\right)=\left[\begin{array}{l}
\left\{\left(x_{f}-x_{i}\right)^{2}+\left(y_{f}-y_{i}\right)^{2}+\left(z_{c}-z_{i}\right)^{2}\right\}^{1 / 2}+v_{1, k} \\
\left\{\left(x_{g}-x_{i}\right)^{2}+\left(y_{g}-y_{i}\right)^{2}+\left(z_{c}-z_{i}\right)^{2}\right\}^{1 / 2}+v_{2, k}
\end{array}\right]
$$

In (7) the subscript, $i$ mean an ultrasonic generator invoked in $k$ time with a mobile robot. Now, we can describe an extended Kalman filter algorithm to estimate state vector of a mobile robot in state equation (5) and observation equation (7) as follows:

$$
\begin{aligned}
& \hat{r}_{k+1}{ }^{-}=f\left(\hat{r}_{k}, u_{k}, 0\right) \\
& P_{k+1}{ }^{-}=A_{k} P_{k} A_{k}^{t}+Q \\
& K_{k}=P_{k}^{-} H_{k}{ }^{t}\left(H_{k} P_{k}{ }^{-} H_{k}{ }^{t}+G\right)^{-1} \\
& \hat{r}_{k}=\hat{r}_{k}{ }^{-}+K_{k}\left(z_{k}-h\left(\hat{r}_{k}{ }^{-}, 0\right)\right) \\
& P_{k}=\left(I-K_{k} H_{k}\right) P_{k}^{-}
\end{aligned}
$$

where, $\hat{r}_{k}^{-}$and $\hat{r}_{k}$ are state vector of an each mobile robot, it is estimate value of a priori and a posteriori with a filter of $r$. $P_{k}^{-}$and $P_{k}$ are error covariance matrix defined as the following formula:

$$
\begin{aligned}
& P_{k}^{-}=E\left[\left(r_{k}-\hat{r}_{k}^{-}\right)\left(r_{k}-\hat{r}_{k}^{-}\right)^{t}\right] \\
& P_{k}=E\left[\left(r_{k}-\hat{r}_{k}\right)\left(r_{k}-\hat{r}_{k}\right)^{t}\right]
\end{aligned}
$$

$$
\begin{aligned}
A_{k} & =\frac{\partial f}{\partial r}\left(\hat{r}_{k}, u_{k}, 0\right) \\
& =\left[\begin{array}{ccc}
1 & 0 & -T_{k} \sin \theta_{k} \\
0 & 1 & T_{k} \cos \theta_{k} \\
0 & 0 & 1
\end{array}\right] \\
H_{k} & =\frac{\partial h}{\partial r}\left(\hat{r}_{k}, 0\right) \\
& =\left[\begin{array}{ccc}
\frac{x_{f}-x_{i}}{D_{f, i}} & \frac{y_{f}-y_{i}}{D_{f, i}} & \frac{C_{f, i}}{D_{f, i}} \\
\frac{x_{g}-x_{n}}{D_{g, i}} & \frac{x_{g}-x_{n}}{D_{g, i}} & \frac{C_{g, i}}{D_{g, i}}
\end{array}\right]
\end{aligned}
$$

where, $C_{f, i}, C_{g, i}$ and $D_{f, i}, D_{g, i}$ are each as follows:

$$
\begin{aligned}
& C_{f, i}=-\left(x_{f}-x_{i}\right) l \sin \theta+\left(y_{f}-y_{i}\right) l \cos \theta \\
& C_{g, i}=\left(x_{g}-x_{i}\right) l \sin \theta-\left(y_{g}-y_{i}\right) l \cos \theta \\
& D_{f, i}=\left\{\left(x_{f}-x_{i}\right)^{2}+\left(y_{f}-y_{i}\right)^{2}+\left(z_{c}-z_{i}\right)^{2}\right\}^{\frac{1}{2}} \\
& D_{g, i}=\left\{\left(x_{g}-x_{i}\right)^{2}+\left(y_{g}-y_{i}\right)^{2}+\left(z_{c}-z_{i}\right)^{2}\right\}^{\frac{1}{2}}
\end{aligned}
$$

We omitted subscript about time of each variable, $k$.

\section{Obstacle confrontation algorithm}

Figure 2 summarizes the ABS system for this research. General situation uses general triangulation technique, but when inaccurate data by obstacle, Twin Beacon Detection or Linear Incremental algorithm takes the place of role of triangulation algorithm. To determine the moment that each algorithm is used to do. It count reliable beacon's data number $\mathrm{b}$ is count in figure 2.

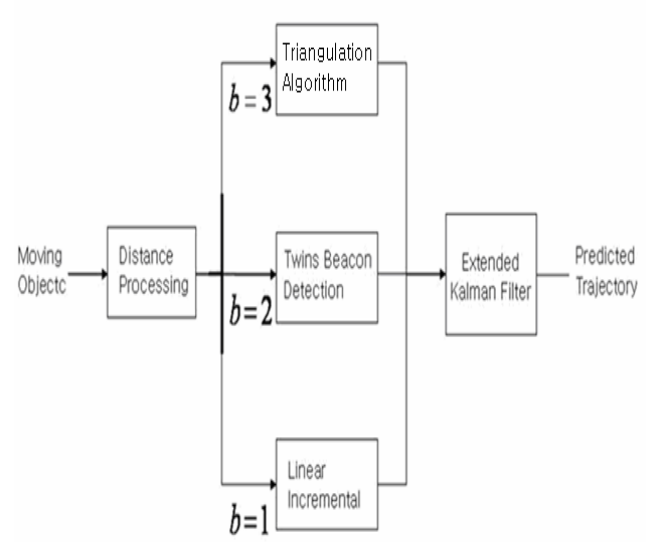

Fig. 2. Countermeasure for obstacle. 


\subsection{Twin Beacon Detection algorithm}

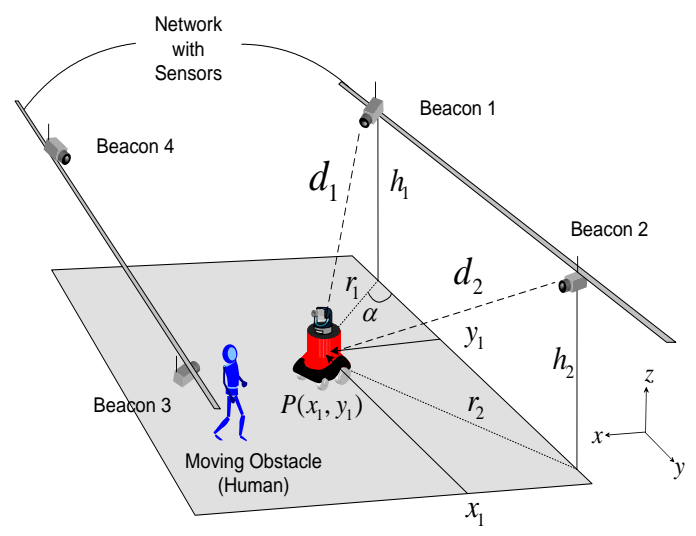

Fig. 3. Countermeasure for obstacle.

$$
\begin{gathered}
x_{1}=r_{1} \cos \alpha \\
y_{1}=\sqrt{r_{1}^{2}-x_{1}^{2}}
\end{gathered}
$$

Twin Beacon Detection algorithm calculates mobile robot position from three beacon through general triangulation technique. But, because indoor environment is complicated, if many obstacles exist, case that miss reliable distance data in beacon more than 3 occurs. This time, This algorithm is able to estimate mobile robot position using 2 beacons that adjoin that do not effect of obstacles [11].

\subsection{Linear incremental algorithm}

Because linear incremental algorithm of indoor environment is complicated, miss reliable distance data in beacon more than 2 if many obstacle exists. In this case, this algorithm is able to estimate mobile robot position using single beacon. Therefore, These algorithm can improve error about outdoor surrounding and the speed if apply algorithm [12-13].

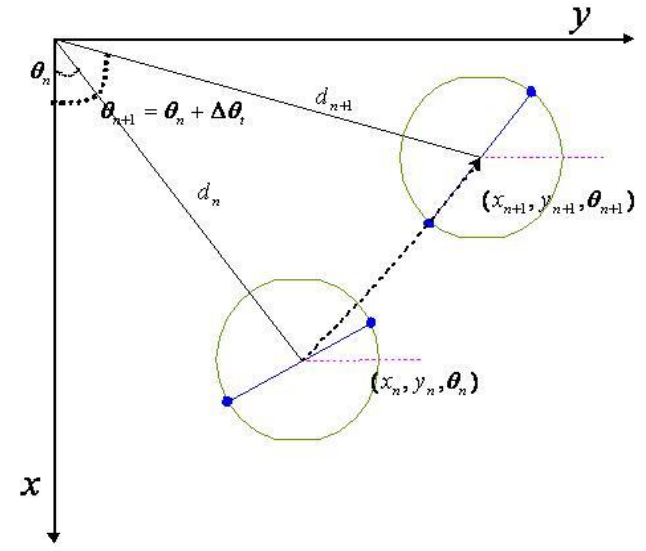

Fig. 4. Linear incremental algorithm.
The first moving robot's coordinate $P=\left[x_{n}, y_{n}, \theta_{n}\right]^{t}$ is already gotten from the beacons more than one, and then its speed is $\dot{P}=\left[\dot{x}_{n}, \dot{y}_{n}\right]$. Besides, its coordinate and speed could be computed as below.

$$
\begin{aligned}
& {\left[\begin{array}{l}
x_{n} \\
y_{n}
\end{array}\right]=\left[\begin{array}{l}
d_{n} \cos \theta_{n} \\
d_{n} \sin \theta_{n}
\end{array}\right]} \\
& {\left[\begin{array}{l}
\dot{x}_{n} \\
\dot{y}_{n}
\end{array}\right]=\left[\begin{array}{cc}
\cos \theta_{n} & -d_{n} \sin \theta_{n} \\
\sin \theta_{n} & d_{n} \cos \theta_{n}
\end{array}\right]\left[\begin{array}{c}
\dot{d} \\
\dot{\theta}
\end{array}\right]} \\
& {\left[\begin{array}{c}
\dot{d} \\
\dot{\theta}
\end{array}\right]=\left[\begin{array}{cc}
\cos \theta_{n} & \sin \theta_{n} \\
-\frac{1}{d_{n}} \sin \theta_{n} & \frac{1}{d_{n}} \cos \theta_{n}
\end{array}\right]\left[\begin{array}{c}
\dot{x}_{n} \\
\dot{y}_{n}
\end{array}\right]}
\end{aligned}
$$

If $\dot{d}$ is replaced with $\Delta d / T$ for the explanation of the discrete time, the formula (18) could be also converted into the formula (19) as below.

$$
\frac{1}{T}\left[\begin{array}{c}
\Delta d \\
\Delta \theta
\end{array}\right]=\left[\begin{array}{cc}
\cos \theta_{n} & \sin \theta_{n} \\
-\frac{1}{d_{n}} \sin \theta_{n} & \frac{1}{d_{n}} \cos \theta_{n}
\end{array}\right]\left[\begin{array}{c}
\dot{x}_{n} \\
\dot{y}_{n}
\end{array}\right]
$$

$\Delta d=d_{n+1}-d_{n}$ could be calculated by inputting the measured distance value. $d_{n+1}=d_{n}+\Delta d, \theta_{n+1}=\theta_{n}+\Delta \theta$ could be calculated by $d_{n+1}$ and $\theta_{n+1}$. Finally we can get the $x_{n+1}$ and $y_{n+1}$ from the formula (16).

\section{Simulations and Experiments}

In experimental environment, the sensor tag is adopted as the sensor for networked beacon, and the tracking of moving objects, such as walking human, and mobile robot, is performed. The environment has a mobile robot as a humanfollowing agent, four beacons which can obtain the situation in the environment, and a wireless LAN and a control unit which communicate suitable information to the robot. Each module is connected through the network communication. Four beacons are used in order to recognize the mobile robot and to generate the control commands. Beacons are placed as shown in Fig. 1.

To use ABS system and extension Kalman filter that propose over to basis, experimented to verify robust characteristics against obstacle algorithm. This system used a manufactured mobile robot using DSP2406 to create trajectory of object that move in this experiment. Manufactured program such as lower part for an experiment. Maximum velocity of an used micro mouse does not pass over $15 \mathrm{~cm} / \mathrm{sec}$. The figure 5 shows the experiment environment and the test bed. The length, width and height of the test bed is respectively $3000 \mathrm{~mm}, 3000 \mathrm{~mm}$ and $2000 \mathrm{~mm}$. 


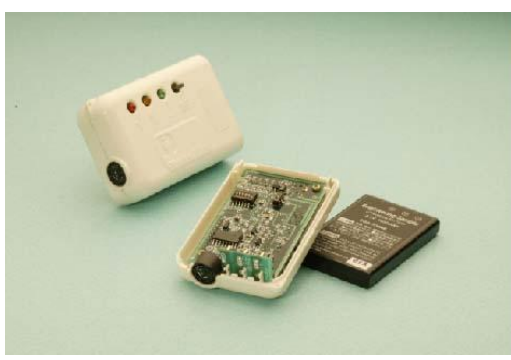

Fig. 5. Sensor tag for Experiments.

\subsection{Twin Beacon Detection algorithm}

Figure 6(a) shows the experimental results of state estimation by the general triangulation technique and by Twin Beacon Detection algorithm for A region have obstacles. Also, by the extension Kalman filter data.

First picture of figure $6(\mathrm{~b})$ shows estimation error by the general triangulation technique and by Twin Beacon Detection algorithm for A region have obstacles. That error increases in A region because general triangulation technique receives noise effect of each beacon data as few than Twin Beacon Detection algorithm. Second picture shows estimation error by extended Kalman filter. It shows that the extended Kalman filter reduce estimation maximum error by about $80 \%$ in A region.

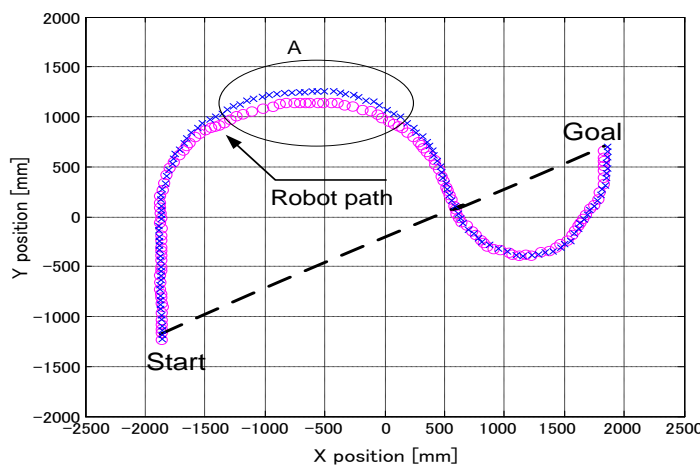

(a) Trajectory of a moving object.
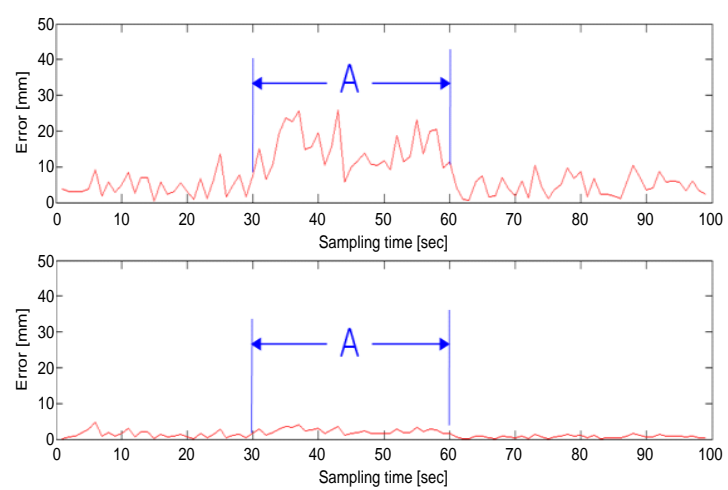

(b) The error between real value and estimate value of a moving object.

Fig. 6. Experiment results of general triangulation technique.

\subsection{Linear Incremental algorithm}

Figure 7 (a) shows the experimental results of state estimation by the general triangulation technique and by Linear Incremental algorithm for B region have obstacles. Also, by the extension Kalman filter data.

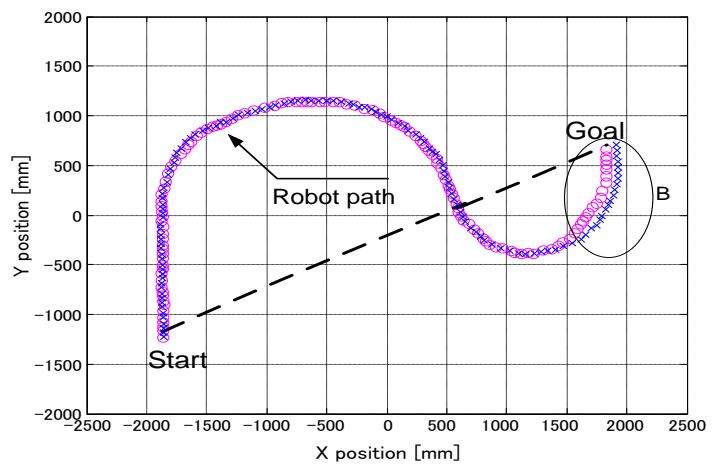

(a) Trajectory of a moving object.
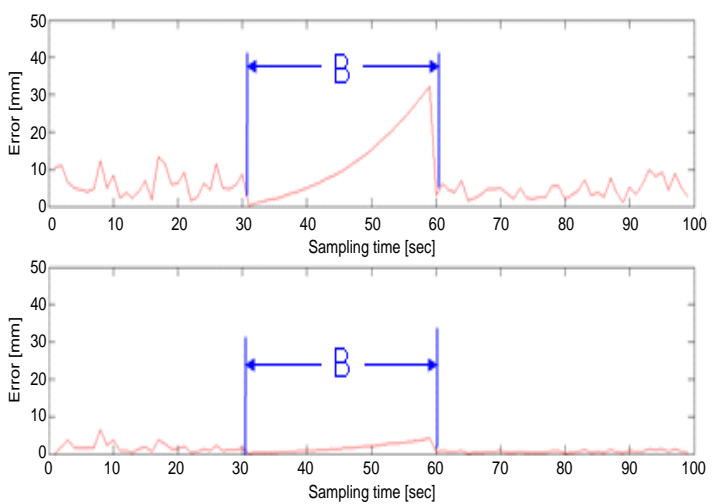

(b) Error between real value and estimate value of a moving object.

Fig. 7. Experimental results of Linear Incremental algorithm.

First picture of figure 7 (b) shows estimation error by the general triangulation technique and by Linear Incremental algorithm for B region have obstacles. That error increases in B region because this region estimated motion information (position of object, direction, speed, acceleration) of mobile robot of verge by standard from stage that is begun, error shows being accumulated. Second picture shows estimation error by extended Kalman filter. The superiority of the proposed algorithm compared with the extended Kalman Filter is demonstrated through real experiments.

\section{Conclusion}

This research proposes a robust absolute position estimate method for obstacles to estimate mobile robot position in door environment. In the schemes Twin Beacon Detection algorithm 
that is method to estimate position by two beacons about problem that could not measure by general triangulation technique method and Liner Incremental algorithm that can estimate position in complicated environment by single beacon. The effectiveness and superiority of the proposed algorithm with extended Kalman Filter was verified through experimental data and comparison.

\section{Acknowledgment}

This research was supported by the 2011 Basic Research Promotion Granted funded by the Dongseo University, and Basic Science Research Program through the National Research Foundation of Korea(NRF) funded by the Ministry of Education, Science and Technology(No. 2010-0021054).

\section{References}

[1] A. Galstyan, B. Krishnamachari, K. Lerman, and S. Pattern, "Distributed online localization in sensor networks using a moving target," in Proc. Int. Symp. Information Processing Sensor Networks (IPSN), pp. 61-70, 2004.

[2] J. Borenstein and L. Feng, "UMBmark-A Method for Measuring, Comparing, and Correcting Dead-reckoning Errors in Mobile Robots," The University of Michigan, Technical Report UM-MEAM-94-22, December, 1994.

[3] Iowa State University GPS page. Web site at http://www.cnde.iastate.edu/gps.html.

[4] T. Arai and E. Nakano, "Development of measuring equipment for location and direction (MELODI) using ultrasonic waves," Trans. ASME, Journal of dynamic systems, Measurement and control, vol 105, pp. 152-156, 1983.

[5] L. Kleeman, "Optimal estimation of position and heading for mobile robots using ultrasonic beacons and Dead-reckoning", Proc. Of IEEE int. Conf. on Robotics and Automaion, pp. 2582-2587, 1992.
[6] Z. Wang; E. Bulut, B.K. Szymanski, "Distributed Target Tracking with Directional Binary Sensor Networks" 2009 IEEE Global Telecommunications Conference, pp. 1- 6, 2009

[7] S. M. Bozic, "Digital and Kalman Filtering," Edward Arnold, 1979.

[8] Greg Welch and Gary Bishop, "An Introduction to the Kalman Filter," 2004.

[9] D. Fox, W. Burgard and S. Thrun, "The dynamic window approach to collision avoidance", IEEE Robotics and Automation Magazine, pp. 23-33, March, 1997.

[10] Soo-Yeong Yi, Jae-Ho Jin, "Self-localization of a Mobile Robot using Global Ultrasonic Sensor System," Journal of Control, Automation and systems Engineering, vol. 9, no 2, pp. 145-151, 2003.

[11] A. So and Y. Ye, "Theory of semidefinite programming for sensor network localization," Mathematical Programming, vol. 109, no. 2, pp. 367-384, 2007.

[12] C. Taylor, A. Rahimi, J. Bachrach, H. Shrobe, and A. Grue, "Simultaneous localization, calibration, and tracking in an ad hoc sensor network," Proc. of IPSN, pp. 27-33, 2006.

[13] S. Sarkka, A. Vehtari, and 1. Lampinen, "Rao-Blackwellized particle filter for multiple target tracking," Information Fusion, vol. 8, no. I, pp. 2-15, 2007.

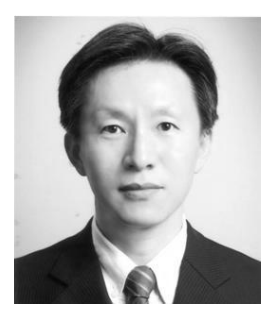

\section{Tae-Seok Jin}

He received the Ph.D. degrees from Pusan National University, Busan, Korea, in 2003, in electronics engineering.

$\mathrm{He}$ is currently an Assistant Professor at DongSeo University. From 2004 to 2005 he was a Postdoctoral Researcher at the Institute of Industrial Science, The University of Tokyo, Japan. His research interests include network sensors fusion, mobile robots, computer vision, and intelligent control. Dr. Jin is a Member of the KIIS, ICROS, and RSJ. 